\title{
Reflection of Singularities of Solutions to Systems of Differential Equations*
}

\author{
MICHAEL E. TAYLOR
}

\section{Introduction}

In this paper we shall examine reflection of singularities of solutions of firstorder equations of the form

$$
\frac{\partial}{\partial y} u=G u
$$

in a region $\Omega$ with boundary $\partial \Omega$ given by $y=0$; say $\Omega=\mathbb{R}+\times \partial \Omega$. Here $G=G(y)=G\left(y, x, D_{x}\right)$ is a smooth one-parameter family of pseudo-differential operators of order one on $\partial \Omega, G(y) \in \operatorname{PS}(1) ; u$ takes on values in a vector space, $\mathbb{C}^{k}$, and $G$ is a $k \times k$ matrix of operators, with principal symbol $G_{1}(y, x, \xi)$, homogeneous of degree one in $\xi$. On the boundary $y=0$, a boundary condition is prescribed:

$$
\beta u(0)=0,
$$

where $\beta \in \mathrm{PS}(0)$ is a pseudo-differential operator of order zero.

We make the assumption that $p(y, x, \eta, \xi)=\operatorname{det}\left(\eta-i G_{1}(y, x, \xi)\right)$ is real and has simple characteristics. Then, as is well known (see [1]), singularities of solutions to (1.1) propagate along the null bicharacteristic strips of $p$ in the interior of $\Omega$. Actually, the reference does not quite apply, since $\partial / \partial y-G$ is not a pseudodifferential operator on $\Omega$ (see the appendix).

Suppose $\left(x_{0}, \xi_{0}\right) \in T^{*}(\partial \Omega)-0$ and that $j$ null-bicharacteristic strips of $p$ pass over $\left(x_{0}, \xi_{0}\right)$. That means there are $j$ real solutions $\eta_{1}, \ldots, \eta_{j}$ of $p\left(0, x_{0}, \eta, \xi_{0}\right)=0$. The associated bicharacteristics $\gamma_{\nu}(t)=(y(t), x(t), \eta(t), \xi(t))$ solve the equations

$$
\begin{aligned}
& \dot{y}=\frac{\partial p}{\partial \eta}, \quad \dot{x}=\nabla_{\xi} p, \\
& \dot{\eta}=-\frac{\partial p}{\partial y}, \quad \dot{\xi}=-\nabla_{x} p,
\end{aligned}
$$

*Results obtained at the Courant Institute of Mathematical Sciences, New York University, with the National Science Foundation, Grant NSF-GP-37069X. Reproduction in whole or in part is permitted for any purpose of the United States Government.

(을 1975 by John Wiley \& Sons, Inc. 
with initial condition $\gamma_{\nu}(0)=\left(0, x_{0}, \eta_{\nu}, \xi_{0}\right)$. We make the assumption that

$$
\text { the bicharacteristc curves intersect } \partial \Omega \text { transversally. }
$$

Thus we are only considering the case of non-glancing rays. This means that $\dot{y}=\partial p / \partial \eta \neq 0$ at $\left(0, x_{0}, \eta_{v}, \xi_{0}\right)$, which implies that all the real zeros $\eta_{1}, \cdots, \eta_{j}$ are simple.

Now, for $(x, \xi)$ in a small conic neighborhood of $\left(x_{0}, \xi_{0}\right)$ and for $y \geqq 0$ small, $p(y, x, \eta, \xi)$ must have exactly $j$ simple roots $\eta_{\nu}(x, \xi)$. This is due to the fact that complex zeros $\eta$ of $p$ must occur in complex conjugate pairs; thus a root cannot wander off the real axis without splitting, which is impossible if it is simple.

It follows that, in a conic neighborhood of $\left(x_{0}, \xi_{0}\right)$ and for $y \geqq 0$ small, $G_{1}(y, x, \xi)$ has $j$ simple pure imaginary eigenvalues $i \lambda_{1}(y, x, \xi), \ldots, i \lambda_{j}(y, x, \xi)$, homogeneous of degree one in $\xi$. Here $\lambda_{\nu}=-\eta_{\nu}$.

The problem we wish to treat is the following: given $u$ which is smooth along the bicharacteristics associated with $\lambda_{1}, \cdots, \lambda_{l}$ lying over $\left(x_{0}, \xi_{0}\right)$, determine when $u$ is smooth along the rest of the bicharacteristics lying over $\left(x_{0}, \xi_{0}\right)$, those associated with $\lambda_{l+1}, \cdots, \lambda_{j}$.

We can define a smooth invertible matrix $U(y, x, \xi)$, homogeneous of degree zero in $\xi$, such that, on a conic neighborhood of $\left(x_{0}, \xi_{0}\right)$, for $y \geqq 0$ small, $U G_{1} U^{-1}$ $=\tilde{G}_{1}$ with

$$
\tilde{G_{1}}=\left(\begin{array}{cccccc}
i \lambda_{1} & & & & \\
& \ddots & & & \\
& & i \lambda_{j} & & \\
& & & E_{+} & \\
& & & & E_{-}
\end{array}\right) .
$$

Here $E_{+}$is a square matrix whose eigenvalues have positive real part, and $E_{-}$one whose eigenvalues have negative real part. If we extend the symbol $\tilde{G}_{1}$ over all $(x, \xi) \in \Omega, 0 \leqq y \leqq y_{0}$, keeping the form (1.4) with the $\lambda$ 's distinct, then (1.1) can be reduced to a simpler equation, in the following fashion.

Let $\psi(x, \xi)$ be a zero-order symbol supported in a small conic neighborhood $U_{0}$ of $\left(x_{0}, \xi_{0}\right)$ and equal to one on a smaller conic neighborhood $U_{1}$. Let

$$
v=U(y, x, D) \psi(x, D) u \text {. }
$$


Then $v$ solves the system

$$
\frac{\partial}{\partial y} v=\tilde{G} v+F
$$

with boundary condition

$$
U \beta U^{-1} v(0)=f
$$

Here $\tilde{G}=U G U^{-1}+U_{y} U^{-1} \in \operatorname{PS}(1)$ has a principal symbol $\tilde{G}_{1}(y, x, \xi)$, and $U^{-1}$ stands for a smooth family of parametrices of $U(y)$. Moreover, $F=U[\psi, G] u$ and $f=U[\beta, \psi] u(0)$ plus smooth functions; we regard these as inhomogeneous terms.

We shall analyze the system (1.6) in the next couple of sections, settling the problem of reflection of singularities in the non-glancing ray case. Section 4 will investigate $H^{s}$ estimates, and then we shall look at some examples, particularly in the case of hyperbolic mixed problems. Finally, in Section 6 we shall consider the problem of obtaining smoothness up to the boundary. We obtain generalizations of the results of Povzner and Sukharevskii [7], and give examples of some grazing-ray flavored phenomena that can occur.

Prior to this work, Andy Majda and Stan Osher had solved such a problem as dealt with in Sections 2-5, for scalar equations, using and extending earlier work of Lax and Nirenberg, as described in [6]: I am grateful to them for conversations which stimulated my interest in this problem.

Warning. In the text, we shall often call a null bicharacteristic strip, which is a curve in $T^{*} \Omega$, a ray. Its projection onto $\Omega$ and, in the case of hyperbolic mixed problems where $\Omega=(0, T) \times \mathcal{\theta}$, its projection onto $\theta$, will also be called a ray. "Smooth along a ray" will mean smooth on a conic neighborhood of the ray in $T^{*} \Omega$.

\section{Uncoupling First-Order Systems}

The system (1.6) is decoupled in its principal part into $j+2$ pieces, since $\tilde{G}_{1}$ has the form (1.4). However, one can expect there to be coupling by terms of order zero. The purpose of this section is to show that we can decouple such a system completely, except for a remainder of order $-\infty$. Wé consider a slightly more general problem. Let $v$ solve the system

$$
\frac{\partial}{\partial y} v=\left(\begin{array}{ll}
F & \\
& E
\end{array}\right) v+A v
$$

where $G=\left(\begin{array}{ll}F & \\ & E\end{array}\right)$ has symbol homogeneous of degree one in $\xi$ and $A=A(y) \in$ $\mathrm{PS}(0)$ has symbol which is an asymptotic sum of terms of order $0,-1,-2$, etc. The assumption we shall make on the symbols $F(y, x, \xi)$ and $E(y, x, \xi)$ is that these 
two matrices, of order $n \times n$ and $m \times m$, respectively, have disjoint sets of eigenvalues, for each $(y, x, \xi) \in \mathbb{R}^{+} \times\left(T^{*}(\partial \Omega)-0\right)$.

We first try for a modest goal, to decouple terms of order zero. Let $w^{(1)}=$ $\left(1+K_{1}\right) v$ with $K_{1} \in \mathrm{PS}(-1)$ to be determined. We have

$$
\begin{aligned}
\frac{\partial}{\partial y} w^{(1)} & =\left(1+K_{1}\right) G\left(1+K_{1}\right)^{-1} w^{(1)}+\left(1+K_{1}\right) A\left(1+K_{1}\right)^{-1} w^{(1)}+\cdots \\
& =G w^{(1)}+\left(K_{1} G-G K_{1}+A\right) w^{(1)}+\cdots,
\end{aligned}
$$

where the remainder involves terms of order at most -1 operating on $w^{(1)}$. We would like to be able to pick $\left(\begin{array}{ll}A_{1} & \\ & A_{2}\end{array}\right)$ and $K_{1}$ such that, on the symbol level,

$$
K_{1} G-G K_{1}+A=\left(\begin{array}{ll}
A_{1} & \\
& A_{2}
\end{array}\right) \text {. }
$$

We take $K_{1}$ to be of the form $\left(\begin{array}{ll}0 & K_{12} \\ K_{21} & 0\end{array}\right)$. This will force solutions of (2.1) to be unique, and hence depend smoothly on parameters. Indeed, if $A=\left(\begin{array}{ll}A_{11} & A_{12} \\ A_{21} & A_{22}\end{array}\right)$, the left side of $(2.1)$ is

$$
\left(\begin{array}{cc}
A_{11} & K_{12} F-E K_{12}+A_{12} \\
K_{21} E-F K_{21}+A_{21} & A_{22}
\end{array}\right) .
$$

Thus we must choose symbols $A_{1}, A_{2}, K_{12}$ and $K_{21}$ such that

$$
\begin{aligned}
A_{1} & =A_{11}, \\
A_{2} & =A_{22}, \\
K_{12} F-E K_{12} & =-A_{12}, \\
K_{21} E-F K_{21} & =-A_{21} .
\end{aligned}
$$

Of these, (2.2) and (2.3) are obvious. We shall show that (2.4) has a unique solution; (2.5) follows similarly.

LeMma 2.1. Let $F \in M_{n \times n}$, the set of matrices of order $n$ by $n$, and $E \in M_{m \times m}$. Define $\phi: M_{n \times m} \rightarrow M_{n \times m}$ by

$$
\phi(T)=T F-E T .
$$

Then $\phi$ is bijective, if $E$ and $F$ have disjoint spectra. 
Proof: It suffices to show $\phi$ is injective; so suppose $T F=E T$. Write $T=\left(\begin{array}{c}T_{1} \\ \vdots \\ \dot{T_{m}}\end{array}\right)$, where each $T_{j}$ is a row of $T$. We may suppose $E$ is in Jordan normal form, say

$$
E=\left(\begin{array}{ccccccc}
\lambda_{1} & & & & & \\
1 & \lambda_{1} & & & & \\
& & \ddots & & & \\
& & 1 & \lambda_{1} & & \\
& & & & \lambda_{2} & \\
& & & & & \ddots
\end{array}\right] .
$$

Assume the first block is $\nu$ by $\nu$. We get

$$
T F=\left(\begin{array}{c}
\lambda_{1} T_{1} \\
T_{1}+\lambda_{1} T_{2} \\
\vdots \\
T_{\nu-1}+\lambda_{1} T_{\nu} \\
\vdots
\end{array}\right),
$$

in particular, $T_{1} F=\lambda_{1} T_{1}$. But $\lambda_{1}$ cannot be an eigenvalue of $F$; so $T_{1}=0$. A simple induction shows that $T=0$.

With such a choice of the symbol of $K_{1}$, we have

$$
\frac{\partial}{\partial y} w^{(1)}=G w^{(1)}+\left(\begin{array}{ll}
A_{1} & \\
& A_{2}
\end{array}\right) w^{(1)}+B w^{(1)}
$$

with $B \in \mathrm{PS}(-1)$. To decouple the part of order -1 , we try $w^{(2)}=\left(1+\mathrm{K}_{2}\right) w^{(1)}$ with $K_{2} \in \mathrm{PS}(-2)$. We get

$$
\begin{aligned}
\frac{\partial}{\partial y} w^{(2)} & =G w^{(2)}+\left(\begin{array}{ll}
A_{1} & \\
& A_{2}
\end{array}\right) w^{(2)}+\left(K_{2} G-G K_{2}+B\right) w^{(2)}+\cdots \\
& =G w^{(2)}+\left(\begin{array}{ll}
A_{1} & \\
& A_{2}
\end{array}\right) w^{(2)}+\left(\begin{array}{ll}
B_{1} & \\
& B_{2}
\end{array}\right) w^{(2)}+\cdots
\end{aligned}
$$


if $K_{2}, B_{1}$, and $B_{2}$ are chosen so that, on the symbol level,

$$
K_{2} G-G K_{2}+B=\left(\begin{array}{ll}
B_{1} & \\
& B_{2}
\end{array}\right) .
$$

This problem was solved just above, so we are in good shape.

From here, you continue, defining $w^{(j)}=\left(1+K_{j}\right) w^{(j-1)}$ with $K_{j} \in \mathrm{PS}(-j)$, decoupling further out along the line; letting $w=(1+K) v$ with $K \in \mathrm{PS}(-1)$ and $1+K$ given by an asymptotic infinite product

$$
1+K \sim \cdots\left(1+K_{2}\right)\left(1+K_{1}\right)
$$

we have

$$
\frac{\partial}{\partial y} w=\left(\begin{array}{ll}
E & \\
& F
\end{array}\right) w+\left(\begin{array}{ll}
\alpha_{1} & \\
& \alpha_{2}
\end{array}\right) w+R w
$$

with $\alpha_{j} \in \mathrm{PS}(0), R=R(y) \in \mathrm{PS}(-\infty)$.

If $v$ solves a boundary problem, it is supposed that $v \in C\left(\left[0, y_{0}\right], H^{s}(\partial \Omega)\right)$ for some $s$; thus $v \in C^{\nu}\left(\left[0, y_{0}\right], H^{s-\nu}(\partial \Omega)\right)$ for $\nu=1,2,3, \cdots$. Consequently, $R w \in C^{\infty}\left(\left[0, y_{0}\right] \times \partial \Omega\right)$. The system is now completely decoupled.

\section{Reflection of Singularities}

Applying the decoupling procedure of Section 2 inductively, we can write $w=(1+K) v$ with $K \in \mathrm{PS}(-1)$ so that $(1.6),(1.7)$ becomes

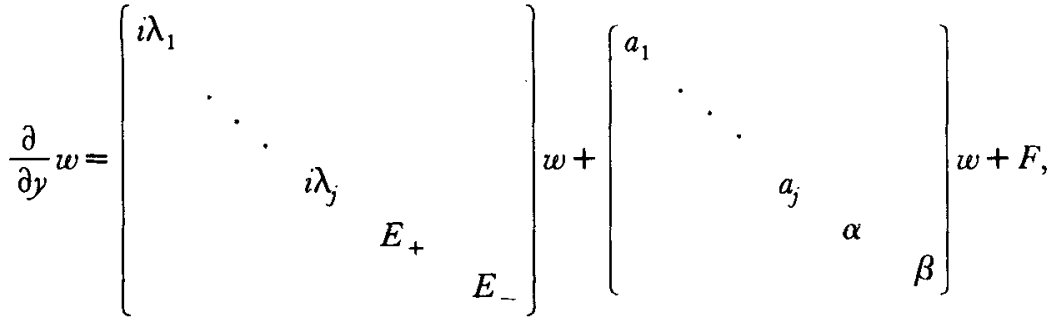

$$
\begin{aligned}
& \gamma w(0)=f,
\end{aligned}
$$

where $a_{\nu}, \alpha, \beta \in \mathrm{PS}(0)$ and $\gamma=(1+K) U \beta U^{-1}(1+K)^{-1} . F=(1+K) U[\psi, G] u$ and $f=(1+K) U[\beta, \psi] u(0)$, plus smooth functions.

Obviously, there is a small conic neighborhood of $\left(x_{0}, \xi_{0}\right)$ disjoint from the wave front set of $f$. We also have the following. Let $\phi(x, \xi)$ be a zero-order symbol supported on the conic neighborhood $U_{1}$ of $\left(x_{0}, \xi_{0}\right)$, where $\psi \equiv 1$.

Lemma 3.1. $\phi(x, D) F \in C^{\infty}\left(\left[0, y_{0}\right] \times \partial \Omega\right)$. 
Proof: Modulo a smooth function,

$$
\begin{aligned}
\phi(x, D) F & =\phi(1+K) U[\psi, G] u \\
& =R(y) u,
\end{aligned}
$$

where $R(y)$ is a smooth one-parameter family of elements of $\mathrm{PS}(-\infty)$. Since $u \in C^{\nu}\left(\left[0, y_{0}\right], H^{s-\nu}(\partial \Omega)\right)$, the desired conclusion is immediate. Write

Tackling the problem of reflection of singularities is now a simple matter.

$$
w=\left(\begin{array}{l}
w^{\mathrm{I}} \\
w^{\mathrm{II}} \\
w^{\mathrm{III}} \\
w^{\mathrm{IV}}
\end{array}\right),
$$

where $w^{\mathrm{I}}$ consists of the first $l$ entries of $w$, corresponding to $i \lambda_{1}, \cdots, i \lambda_{l}, w^{\mathrm{II}}$ consists of the next $j-l$ entries, corresponding to $i \lambda_{l+1}, \cdots, i \lambda_{j}, w^{\mathrm{III}}$ is acted on by $E_{+}$, and $w^{\text {IV }}$ is acted on by $E_{-}$.

Since propagation of singularities inside $\Omega$ is well understood, we take $y_{0}$ to be so small that the bicharacteristic strips lying over $\left(x_{0}, \xi_{0}\right)$ at $y=0$ all lie over $U_{1}$ for $y \in\left[0, y_{0}\right]$. Suppose now that the solution $u$ to $(1.1)$ is smooth near the bicharacteristic strips associated with $\lambda_{1}, \cdots, \lambda_{l}$. Then so is $w^{\mathrm{I}}$. But $w^{\mathrm{I}}$ satisfies a hyperbolic equation all by itself, so

$$
w^{\mathrm{I}}(0) \text { is smooth near }\left(x_{0}, \xi_{0}\right) \text {. }
$$

In addition, $w^{\mathrm{III}}$ solves a backward parabolic equation, so

$$
w^{\mathrm{III}}(0) \text { is smooth near }\left(x_{0}, \xi_{0}\right) \text {. }
$$

Now $w^{\mathrm{II}}$ solves a hyperbolic equation and $w^{\mathrm{IV}}$ a forward parabolic equation; so if the system of pseudo-differential equations (3.2)-(3.4) leads to smoothness of $w^{\mathrm{II}}(0)$, we get $w$ smooth near $\left(x_{0}, \xi_{0}\right)$ for $y \in\left(0, y_{0}\right]$, and, if it also leads to smoothness of $w^{\mathrm{IV}}(0)$, we get $w$ smooth near $\left(x_{0}, \xi_{0}\right)$ for $y \in\left[0, y_{0}\right]$.

We now translate back to the systems $(1.1),(1.2)$ and state our main theorem. Let $P^{\mathrm{I}} u=U^{-1}(1+K)^{-1} w^{\mathrm{I}}, P^{\mathrm{II}} u=U^{-1}(1+K)^{-1} w^{\mathrm{II}}, P^{\mathrm{III}} u=U^{-1}(1+K)^{-1} w^{\mathrm{III}}$. Thus $P^{\mathrm{I}}=P^{\mathrm{I}}\left(y, x, D_{x}\right) \in \mathrm{PS}(0)$, as are $P^{\mathrm{II}}$ and $P^{\mathrm{III}}$.

THEOREM 3.2. Let the solution to (1.1), (1.2) be smooth along those bicharacteristics lying over $\left(x_{0}, \xi_{0}\right) \in T^{*}(\partial \Omega) \backslash 0$ associated with $\lambda_{1}, \cdots, \lambda_{l}$. If the system of pseudo-differential 
operators

$$
P^{\mathrm{I}} u(0)=f, \quad P^{\mathrm{III}} u(0)=g, \quad \beta u(0)=h \quad \text { smooth near }\left(x_{0}, \xi_{0}\right)
$$

implies smoothness of $P^{\mathrm{II}} u(0)$ near $\left(x_{0}, \xi_{0}\right)$, then $u$ is smooth along the rest of the bicharacteristic strips over $\left(x_{0}, \xi_{0}\right)$, in the interior of $\Omega$. If the system (3.5) has the regularity property for $u(0)$ near $\left(x_{0}, \xi_{0}\right)$, then in addition $\phi(x, D) u$ is smooth on $\left[0, y_{0}\right) \times \partial \Omega$, where the symbol $\phi(x, \xi)$ is supported in the small neighborhood $U_{1}$ of $\left(x_{0}, \xi_{0}\right)$.

All this has been proved for $w(y)=(1+K) U \psi(x, D) u$, and it is necessary only to apply a smooth family of parametrices to $(1+K) U \psi(x, D)$, which is elliptic near $\left(x_{0}, \xi_{0}\right)$ for $y \in\left[0, y_{0}\right]$.

The principal symbols of $P^{\mathrm{I}}$ and $P^{\mathrm{III}}$ are simply the projections onto the linear span in $\mathbb{C}^{k}$ of the eigenspaces associated with the eigenvalues $i \lambda_{1}, \cdots, i \lambda_{l}$ of $G_{1}(0, x, \xi)$ and onto the linear span of the generalized eigenspaces corresponding to eigenvalues of $G_{1}(0, x, \xi)$ with positive real part, respectively. Thus ellipticity of (3.5) near $\left(x_{0}, \xi_{0}\right)$ is easy to investigate. If one is to investigate hypoellipticity of (3.5), with loss of at least one derivative, some untangling is required. Lower order terms in (1.1) make their presence felt in (3.5) via the operator $K$. We shall not make a more explicit analysis of hypoellipticity here. A number of examples in the scalar case are given in [5].

The technique we have used of breaking our system up into a forward evolution and backward evolution part is familiar in the theory of elliptic boundary value problems and was introduced by Calderon; see [8].

\section{4. $\mathrm{H}^{\mathrm{s}}$ Theory}

We restrict our attention to $\left[0, y_{0}\right] \times \partial \Omega$, where $y_{0}$ is chosen small, as before.

Suppose the solution $u$ to (1.1), (1.2) has the following smoothness along the null bicharacteristics $\gamma_{\nu}$ lying over $\left(x_{0}, \xi_{0}\right) \in T^{*}(\partial \Omega) \backslash 0$ associated with the eigenvalues $i \lambda_{\nu}, \nu=1, \cdots, l: u \in H^{s}$ in a conic neighborhood $\mathcal{O}_{\nu}$ of the $\gamma_{\nu}$, for $y \in\left(y_{1}, y_{2}\right)$ with $0<y_{1}<y_{2} \geqq y_{0}$. Now $w=(1+K(y)) U(y) \psi\left(x, D_{x}\right) u$ is obtained from $u$ by applying an operator of order zero. This operator is not a pseudo-differential operator on $\Omega$ because its symbol,

$$
\sigma(y, x, \eta, \xi)=(1+K(y, x, \xi)) U(u, x, \xi) \psi(x, \xi),
$$

is singular at $\xi=0$. However, the conic subsets of $T^{*} \Omega \backslash 0$ we are considering here are disjoint from this set, so $\sigma\left(y, x, D_{x}\right)$ behaves like a pseudo-differential operator there. Thus $w \in H^{s}$ on $\Theta_{\nu}, \nu=1, \cdots, l$. Propagating this down close to $\partial \Omega$ according to [1], Proposition 3.5.1, we see that for $y \in\left(0, y_{1}\right), y_{1}$ small, $w \in H_{\text {loc }}^{s}$ on $\Upsilon_{\nu}$, the subset of $T^{*}\left(\left(0, y_{1}\right) \times \partial \Omega\right) \backslash 0$ lying over a conic neighborhood $U_{1}$ of $\left(x_{0}, \xi_{0}\right)$. In particular we apply this to $w^{I}$, which solves a hyperbolic equation. 
Lemma 4.1. $w^{\mathrm{I}} \in C\left(\left(0, y_{1}\right), H_{U_{1}}^{s}(\partial \Omega)\right)$. Here $H_{\Gamma}^{s}(M)$ denotes the space of distributions on $M$ which belong to $H^{s}$ on the conic subset $\Gamma$ of $T^{*} M \backslash 0$.

Proof: First suppose $s=0$. That $w^{\mathrm{I}}(y) \in H_{U_{1}}^{0}(\partial \Omega)$ and is continuous in $y$ (say for $y<\alpha y_{1}$ ) follows from the formula

$$
w^{\mathrm{I}}(y)=\frac{1}{y_{1}-\alpha y_{1}} \int_{\alpha y_{1}}^{y_{1}} E(y, \tau) w^{\mathrm{I}}(\tau) d \tau
$$

where $E(y, \tau)$ is the solution operator for the hyperbolic equation

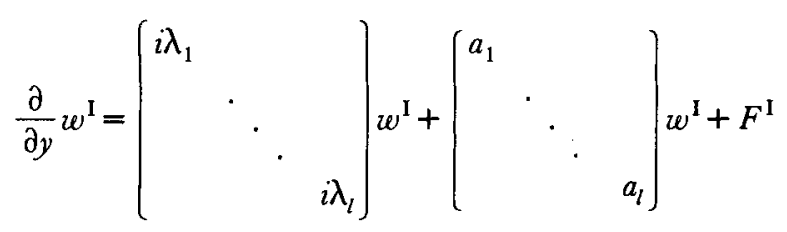

taking data at "time" $\tau$ into data at "time" $y$. This would also work for $s \geqq 0$; but for general $s$ we make the transformation $\omega^{\mathrm{I}}=\Lambda^{s} w^{\mathrm{I}}$, where $\Lambda^{s}$ is an elliptic operator of order $s$ on $\partial \Omega$, and apply the $s=0$ result to $\omega^{\mathbf{I}}$.

From Lemma 4.1 we conclude that $w^{\mathrm{I}}(0) \in H^{s}$ near $\left(x_{0}, \xi_{0}\right)$, since $w^{1}$ solves (4.1). It is now easy to put the main theorem of the previous section in an $H^{s}$ setting.

TheOrem 4.2. Assume the solution u to (1.1), (1.2) belongs locally to $H^{s}$ in a neighborhood of the null bicharacteristic strips $\gamma_{1}, \cdots, \gamma_{l}$. If the system of pseudo-differential equations

$$
P^{\mathrm{I}} u(0), P^{\mathrm{III}} u(0), \beta u(0) \in H^{s} \text { near }\left(x_{0}, \xi_{0}\right)
$$

implies that $P^{\mathrm{II}} u(0) \in H^{\sigma}$ near $\left(x_{0}, \xi_{0}\right)$, then $u \in H^{\sigma}$ in a neighborhood of the rest of the null bicharacteristic strips passing over $\left(x_{0}, \xi_{0}\right)$. If in addition the system (4.2) implies that $P^{\mathrm{IV}} u(0) \in H^{\sigma-1 / 2}$ on a neighborhood $U_{1}$ of $\left(x_{0}, \xi_{0}\right)$, then $\phi(x, D) u \in H^{o}\left(\left[0, y_{0}\right] \times \partial \Omega\right)$, where $\phi \in S_{1,0}^{0}$ is supported in $U_{1}$.

Proof: The regularity properties above imply, respectively, that $w^{\mathrm{II}}(0) \in H^{\sigma}$ and $w^{\mathrm{IV}}(0) \in H^{\sigma}$ near $\left(x_{0}, \xi_{0}\right)$, from which the associated regularity of $w$ is immediate.

Now since $w(y)=V(y) u(y)$ with $V \in \operatorname{PS}(0)$ elliptic near $\left(x_{0}, \xi_{0}\right)$ for each $y \in\left[0, y_{0}\right]$, letting $W(y) \in \operatorname{PS}(0)$ be a parametrix for $V$ near $\left(x_{0}, \xi_{0}\right)$ depending smoothly on $y$, we have

$$
u(y)=W(y) w(y)+R(y) u(y)
$$

where the symbol of $R(y)$ is of order $-\infty$ in a conic neighborhood of $\left(x_{0}, \xi_{0}\right)$. 
From the fact that $W(y)$ and $R(y)$ act on $w$ like pseudo-differential operators on $\left(0, y_{0}\right) \times \partial \Omega$, follows the stated regularity of $u$ on the interior. The formula

$$
\phi(x, D) u=\phi(x, D) W(y) w(y)+\phi(x, D) R(y) u(y)
$$

yields the final assertion of the theorem.

The above regularity assumptions on (4.2) are also necessary for the conclusions on $u$. For $w^{\text {II }}$ this is immediate; $w^{\text {II }}$ solves a hyperbolic equation. The necessity for $w^{\mathrm{IV}}$ is well known in the elliptic theory. The case $s=\sigma$ is the case of no loss of derivatives for the solution $u$. It is interesting that something less than ellipticity of the system (4.2) is needed here. Since ellipticity is the "natural" hypothesis to make and the one we shall see arising in examples, this is slightly mysterious.

\section{Examples}

We look now at certain examples of systems where the results of Theorems 3.2 and 4.2 apply. In all of our examples, the system (4.2) will be elliptic. The first example we consider is that of a strictly hyperbolic system, of order $k$,

$$
\frac{\partial}{\partial t} u=H u=A \frac{\partial}{\partial y} u+\sum_{j=1}^{n} B_{j} \frac{\partial}{\partial x_{j}} u+C u,
$$

where $u=u(t, x, y)$ is defined on $\Omega=\mathbb{R} \times \hat{\theta}=\mathbb{R} \times \partial \theta \times \mathbb{R}^{+} ; \partial \Omega=\mathbb{R} \times \partial \theta$. We assign a boundary condition

$$
\beta u=0 \text { on } \partial \Omega
$$

which we assume satisfies Kreiss' condition for well-posedness, described in [4]. We assume (5.1) is non-characteristic, i.e., $A^{-1}$ exists.

Over $\zeta_{0}=\left(t_{0}, x_{0}, \tau_{0}, \xi_{0}\right) \in T^{*}(\partial \Omega) \backslash 0$ pass a number of null bicharacteristic strips, say $\gamma_{1}, \cdots, \gamma_{j}$, which we assume to be non-glancing. Suppose $\gamma_{1}, \cdots, \gamma_{l}$ go off into $\Omega$ in the negative $t$ direction $(i<0)$ and $\gamma_{l+1}, \cdots, \gamma_{j}$ go off into $\Omega$ in the positive $t$ direction $(i>0)$. The convention in both cases is $\dot{y}>0$.

Proposition 5.1. If $u \in H^{s}$ along $\gamma_{1}, \cdots, \gamma_{l}$, then $u \in H^{s}$ along $\gamma_{i+1}, \cdots, \gamma_{j}$ (at least until they hit $\partial \Omega$ again).

Proof: We verify that the system (4.2) is elliptic. Our system is

$$
\begin{aligned}
\frac{\partial}{\partial y} u & =A^{-1}\left[\frac{\partial}{\partial t} u-\sum_{j=1}^{n} B_{j} \frac{\partial}{\partial x_{j}} u-C u\right) \\
& =G u
\end{aligned}
$$


where the principal symbol of $G$ is

$$
G_{1}(t, x, \tau, \xi)=i A^{-1}\left(\tau-\sum_{j=1}^{n} B_{j} \xi_{j}\right)
$$

which has near $\zeta_{0}$ pure imaginary eigenvalues $i \lambda_{1}, \cdots, i \lambda_{j}$ to which correspond the bicharacteristic strips $\gamma_{1}, \cdots, \gamma_{j}$, and $k-j$ eigenvalues with nonzero real part. If $E^{\mathrm{I}}\left(\zeta_{0}\right)$ is the span of the eigenvectors of $G_{1}$ corresponding to $i \lambda_{1}, \cdots, i \lambda_{l}$ and $E^{\mathrm{II}}$, $E^{\mathrm{III}}$, and $E^{\mathrm{IV}}$ are defined in an analogous fashion, then ellipticity of (4.2) is equivalent to the condition: for $\omega \in \operatorname{ker} \beta_{0}$, the projection of $\omega$ on $E^{\mathrm{I}}$ and $E^{\mathrm{III}}$ determines $\omega \in \mathbb{C}^{k}$ (where $\beta_{0}$ is the symbol of $\beta$ ). This is equivalent to saying

$$
E^{\mathrm{II}} \oplus E^{\mathrm{IV}} \cap \operatorname{ker} \beta_{0}=\{0\} .
$$

Let us compare this to the Kreiss condition. Take $\eta<0$ and consider (at $\left.(t, x, \tau, \xi)=\zeta_{0}\right)$

$$
M(\eta)=i A^{-1}\left(\tau+i \eta-\sum_{j=0}^{n} B_{j} \xi_{j}\right) .
$$

All the eigenvalues of this matrix have nonzero real part, and if $V(\eta)$ denotes the linear span of the generalized eigenvectors of $M(\eta)$ with negative real part, the Kreiss condition implies

$$
V(\eta) \text { is bounded away from } \operatorname{ker} \beta_{0} \text { as } \eta \uparrow 0 \text {. }
$$

To relate (5.3) and (5.4), note that along a null bicharacteristic strip $\gamma_{\nu}, i=$ $-\partial \lambda_{\nu} / \partial \tau=-(1 / i) \partial \lambda_{\nu} / \partial \eta$; thus if $\dot{i}<0$, we must have $\mathscr{R} e i \lambda_{\nu}>0$ for small $\eta<0$, while if $i>0$, we have $R e i \lambda_{v}<0$ for small $\eta<0$. It follows that $V(\eta)$ approaches $E^{\mathrm{II}} \oplus E^{\mathrm{IV}}$ as $\eta \uparrow 0$, so (5.4) $\Rightarrow(5.3)$.

To deduce ellipticity for the system (4.2), relation (5.4) had only to hold in those directions where $G_{1}$ can be decoupled, since we are only obtaining results for non-glancing rays. For our problem, the rest of the directions (the "characteristic variety", on which Kreiss had to work so hard) plays no role, though it would be expected to do so if one were to treat glancing rays. Thus Proposition 5.1 can be generalized to many problems which do not satisfy Kreiss' conditions, such as the Neumann boundary condition for the operator $\partial^{2} / \partial t^{2}-\Delta$ (which is dealt with in [5]).

Another class of examples is given by higher-order scalar equations

$$
\begin{gathered}
\left(D_{y}^{m}+\sum_{j=0}^{m-1} \sum_{|\alpha|+j=m} a_{\alpha, j} D_{y}^{j} D_{x}^{\alpha}\right) f=0, \\
B f=0 \text { at } y=0 .
\end{gathered}
$$


This can be converted into a first-order equation for an $m$-vector-valued function $u$ with components $u_{j}=D_{y}^{j} \Lambda^{-j} f, j=0, \cdots, m-1$, where $\Lambda$ is a pseudo-differential operator on $\partial \Omega$ with principal symbol $|\xi|$. When Theorem 4.2 is applied to the resulting system, the system (4.2) can be analyzed in the same fashion as are elliptic boundary value problems (see [11], Ghapter V, especially Section 3). We shall not carry this out, since the scalar case is examined in detail, by another method, in [5]. Higher-order systems can also be converted to first-order systems and analyzed.

Next we examine reflection of singularities for solutions to Maxwell's equations in a vacuum region $\Re$ bounded by a perfect conductor, in the case of non-glancing rays. The electric and magnetic fields, $E$ and $B$, solve eight equations:

$$
\begin{aligned}
\frac{\partial}{\partial t} E-\operatorname{curl} B & =0, \\
\frac{\partial}{\partial t} B+\operatorname{curl} E & =0, \\
\operatorname{div} E & =0, \\
\operatorname{div} B & =0,
\end{aligned}
$$

and, on the boundary of the conductor, $E$ is normal and $B$ is tangential. It is convenient to write boundary value problems for $E$ and $B$, separately, as follows:

$$
\begin{aligned}
\frac{\partial^{2}}{\partial t^{2}} E-\Delta E & =0, \\
\operatorname{div} E & =0 \text { and } E \times \nu=0 \text { on } \partial \Re, \\
\frac{\partial^{2}}{\partial t^{2}} B-\Delta B & =0, \\
B \cdot \nu & =0 \text { and } \nu \times \operatorname{curl} B=0 \text { on } \partial \Re .
\end{aligned}
$$

We need only analyze reflection of singularities for equations (5.9) and (5.10), with their associated boundary conditions. We may regard (5.5)-(5.8) as compatibility conditions which must be satisfied by the Cauchy data, at time $t_{0}$, and which then are automatically satisfied for all $t$.

One can convert (5.9) and (5.10) to first-order systems, but indeed the results of [5] apply, since the wave operator is scalar, even though it is acting on vector-valued quantities whose boundary values are coupled. Either way, it is easily verified that reflection of singularities results, analogous to those of Proposition 5.1, hold. 
Another interesting example is provided by a transmission problem for the wave equation with different sound speeds in two adjacent regions, $\Omega_{1}$ and $\Omega_{2}$, separated by a smooth surface $S$. For example one can take $\left(D_{t}^{2}-\Delta\right) u=0$ on $\Omega_{1}$ and $\left(D_{t}{ }^{2} / c^{2}-\Delta+a \partial / \partial t\right) u=0$ on $\Omega_{2}$ while the values of $u$ and its normal derivatives match up on the two sides of $S$.

This can be converted into a boundary value problem by identifying $\Omega_{2}$ with $\Omega_{1}$, smoothly in a neighborhood of $S$. We are led to a second-order system of the form

$$
\begin{aligned}
& \left(\frac{\partial^{2}}{\partial t^{2}}-A_{1}\left(x, D_{x}\right)\right) u=0, \\
& \left(\frac{\partial^{2}}{\partial t^{2}}-A_{2}\left(x, D_{x}\right)\right) v=0,
\end{aligned}
$$

with boundary conditions

$$
\begin{gathered}
u=v \quad \text { on } S, \\
\frac{\partial}{\partial y} u=-\frac{\partial}{\partial y} v \quad \text { on } S,
\end{gathered}
$$

where $y$ is the normal coordinate to $S, A_{j}\left(x, D_{x}\right)$ are second-order differential operators with principal symbols $A_{j}(x, \xi) \leqq-C_{1}|\xi|^{2}$, for some $C_{1}>0$. The boundary value problem (5.12) does not satisfy the Kreiss condition, but the remark following the proof of Proposition 5.1 can be applied to show that if $\left(\begin{array}{l}u \\ v\end{array}\right)$ is smooth along the rays coming in from negative time and lying over $p_{0} \in T^{*}(\mathbb{R} \times S) \backslash 0$, both assumed to be non-grazing, then $\left(\begin{array}{l}u \\ v\end{array}\right)$ is smooth along the corresponding rays issuing forth into positive time. (Note that over any $p_{0} \in \bar{T}^{*}$ $(\mathbb{R} \times S) \backslash 0$ pass either 0,2 , or 4 rays. $)$

If only two rays pass over $p_{0}$, they must be incident and reflected rays for the slow speed region $\left(\Omega_{1}\right.$ if $\left.c>1\right)$. This is the case of total internal reflection. If four rays pass over $p_{0}$, all non-grazing, then one can check that smoothness over any pair leads to smoothness over the other pair, with no loss of derivatives. From this it follows that if $u$ is smooth along one ray coming in from negative time and belongs to $H^{s}$ along the other ray, but not to $H^{s+\epsilon}$, then along the two rays over $p_{0}$ going into positive time, $u$ belongs to $H^{s}$ but not to $H^{s+\epsilon}$.

It is possible that one pair of rays passing over $p_{0}$ is non-grazing and the other pair is grazing. The theorems we have proved do not cover this case, which will be discussed further in the next section.

An interesting contrast occurs if we take $c \equiv 1$. Then the transmission problem is merely the wave equation with a discontinuous "friction" term $a \partial / \partial t$. In this case, if $u \in H^{s}$ along one incident ray and smooth along the other, then $u \in H^{s}$ 
along the transmitted ray, but $u \in H^{s+1}$ along the reflected ray, i.e., reflected waves are relatively weak.

\section{Smoothness up to the Boundary}

In this section we consider solutions to a hyperbolic mixed problem on $\Omega=\mathcal{O} \times(0, T)$. Our purpose is to give a precise characterization of the singularities of such solutions $u$, for $t \in(0, T)$, given that, for $t \in(0, \tau), u$ is only singular on rays that pass over points $p_{0} \in T^{*}(\partial \Omega) \backslash 0$ with the property that all null bicharacteristics passing over this point are non-grazing, and that the reflected rays have a similar property, at least up to time $T$. In fact, we suppose that all such rays are bounded away from grazing directions. We shall refer to this as the "non-grazing hypothesis".

For convenience we shall suppose that $\overline{\mathcal{\theta}}$ is compact, although this restriction may be relaxed by finite propagation speed.

Theorem 6.1. Suppose that $u \in C\left([0, T], H^{s}(\theta)\right)$ solves a Kreiss well posed mixed problem for a first-order strictly hyperbolic system $(\partial / \partial t-H) u=0$, with $\beta u=0$ on $\partial \Omega$. Suppose $u$ is singular only along a set of null bicharacteristic curves $S$ and is smooth near $\partial \theta$ for $t \in(0, \tau)$, and satisfies the non-grazing hypothesis. Then we have the following conclusions:

(a) In the interior of $\Omega, u$ is singular only along the set of rays $\tilde{S}$ arising from $S$ upon propagation and reflection at $\partial \Omega$ (each ray hitting $\partial \Omega$ giving rise to perhaps several reflected rays).

(b) Take $p \in \partial \Omega$ and let $S_{p}$ be the image in $T^{*}(\partial \Omega)$ of $\tilde{S}$ intersected with a small neighborhood of $p$. (Think of a small neighborhood of $\partial \Omega$ as $\left.\left(0, y_{0}\right) \times \partial \Omega\right)$.) Then $\psi\left(x, t, D_{x, t}\right) u \in C^{\infty}$ on $\left[0, y_{0}\right) \times \partial \Omega$ for any $\psi \in \mathrm{PS}(0, \partial \Omega)$ with symbol supported on $(x, t)$ close to $p$ and vanishing in a conical neighborhood of $S_{p}$. (It need not vanish on the "characteristic variety".)

In particular, if no element of $\tilde{S}$ lies over $p \in \partial \Omega, u$ is smooth in a neighborhood of $p$.

Proof: What we shall do is construct a $v \in C\left([0, T], H^{s}(\theta)\right)$ with the properties:

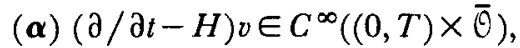

( $\beta$ ) $\beta v \in C^{\infty}(\partial \Omega)$,

$(\gamma) u-v \in C^{\infty}((0, \tau) \times \bar{\theta})$

(ठ) $v$ obviously satisfies conclusions (a) and (b).

From $(\boldsymbol{\alpha})-(\boldsymbol{\gamma})$ it follows that $u-v \in C^{\infty}((0, T) \times \overline{\mathcal{Q}})$. This is easy to see if $H$ is elliptic and the boundary conditions are coercive. In general it follows from [10]. Then the theorem follows from $(\boldsymbol{\delta})$.

Near $\partial \Omega$, the equation for $u$ can be written as a boundary value problem for a system of the form $(\partial / \partial y-G) u=0$, with $\partial \Omega=\{(y, x, t): y=0\}$. (We set $z=(x, t)$.) We can cover the complement of the characteristic variety in $T^{*}(\partial \Omega) \backslash 0^{\circ}$ with 
conic open sets $\mathfrak{A}_{\alpha}$ with the property that the symbol of $G$ can be put in the form (1.4) on each $\mathfrak{A}_{\alpha}$ for $y \in\left[0, y_{1}\right]$. The non-glancing hypothesis in $u$ allows us to write $u$ as a finite sum $\sum_{v=1}^{r} u_{p}$ with the property that, for fixed $j$, all the nullbicharacteristic strips carrying $\mathrm{WF}\left(u_{\nu}\right)$ on $(0, \tau) \times \Theta$ propagate along to $\partial \Omega$ along a very narrow conic neighborhood of a single ray and pass over some $\Sigma \subset T^{*}(\partial \Omega) \backslash 0$ with $\Sigma$ contained in some one $\mathfrak{A}_{\alpha}$, say $\mathfrak{A}_{1}$. We may suppose $(x, t, \zeta) \in \Sigma$ only if $t \in\left(\tau_{1}, \tau_{1}+\frac{1}{2} \epsilon\right)$ and that for $t \in\left(\tau_{1}, \tau_{2}\right)$ all the reflected rays lie in the interior of $\Omega$. Each such ray hitting $\partial \Omega$ gives rise to, say, $j-l$ reflected rays, and furthermore we may assume that each bunch of reflected rays so produced enjoys a similar property, and so on, up to time $T$. We do this in order to localize the construction of $v$.

The behavior of $\operatorname{WF}\left(u_{\nu}\right)$ for $t \in\left(0, \tau_{1}\right)$ is clear. What we now do is construct $v_{\nu}$ for $t \in\left(0, \tau_{1}+\epsilon\right)$, with properties analogous to $(\boldsymbol{\alpha})-(\boldsymbol{\delta})$. This will completely describe the singularities of $u_{\nu}$ for $t \in\left(0, \tau_{2}\right)$, and repeating such a procedure will get us up to time $T$, so the proof will be complete.

Recall that if $\psi(z, \zeta)$ is a zero-order symbol supported in $\mathfrak{A}_{1}$ and equal to one in a conic neighborhood $\tilde{\mathfrak{A}}_{1}$ of $\Sigma$, then the construction of Sections 1-3 yields equation (3.1) for $w=(1+K) U \psi\left(z, D_{z}\right) u_{\nu}$, with boundary condition (3.2). We suppose that $\lambda_{1}, \cdots, \lambda_{l}$ represents incoming modes, i.e., $i=-\partial \lambda_{\nu} / \partial \tau<0$, and $\lambda_{l+1}, \cdots, \lambda_{j}$ represent reflected waves. Thus, for $t<\tau_{1}$, WF $(w)$ is contained in the null bicharacteristic strips associated with $\lambda_{1}, \cdots, \lambda_{l}$, passing over $\Sigma$.

Recall that we are writing a small neighborhood of $\partial \Omega$ as $\left[0, y_{1}\right] \times \partial \Omega$. We may suppose that $y_{1}$ is so small that if $(y, x, t, \eta, \xi, \tau) \in \tilde{S}, t<\tau_{2}$, and if $y \leqq y_{1}$, then $t \in\left(\tau_{1}-\epsilon, \tau_{1}+\epsilon\right)$ and $(x, t, \xi, \tau) \in \tilde{\mathfrak{A}}_{1}$. Also suppose $y \geqq \frac{1}{2} y_{1}, t<\tau_{1}+\epsilon \Rightarrow t<\tau_{1}$.

We define an approximate solution $W=\left[\begin{array}{l}W^{\mathrm{I}} \\ W^{\mathrm{II}} \\ W^{\mathrm{III}} \\ W^{\mathrm{IV}}\end{array}\right]$ as follows. For $y \in\left[\frac{1}{2} y_{1}, y_{1}\right]$, let $W^{\mathrm{I}}$ be any smooth extension of $w^{\mathrm{I}}$ for $t \in\left(0, \tau_{1}\right)$ to $t \in(0, T)$. Then

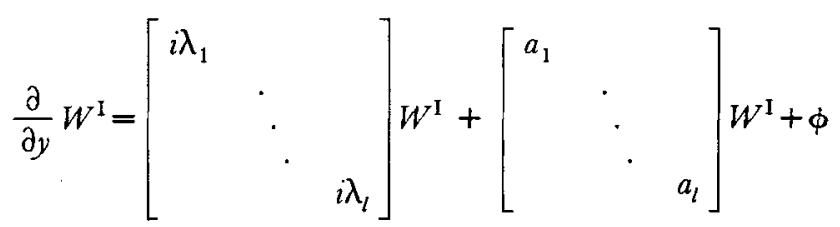

with $\phi$ smooth, for $y \in\left[\frac{1}{2} y_{1}, y_{1}\right]$. Now take any smooth extension of $\phi$ for $y \in\left[0, \frac{1}{2} y_{1}\right]$ and extend $W^{\mathrm{I}}$ to solve (6.1). Let $W^{\mathrm{III}}=0$. We complete the prescription of $W$ by 
requiring that it satisfy

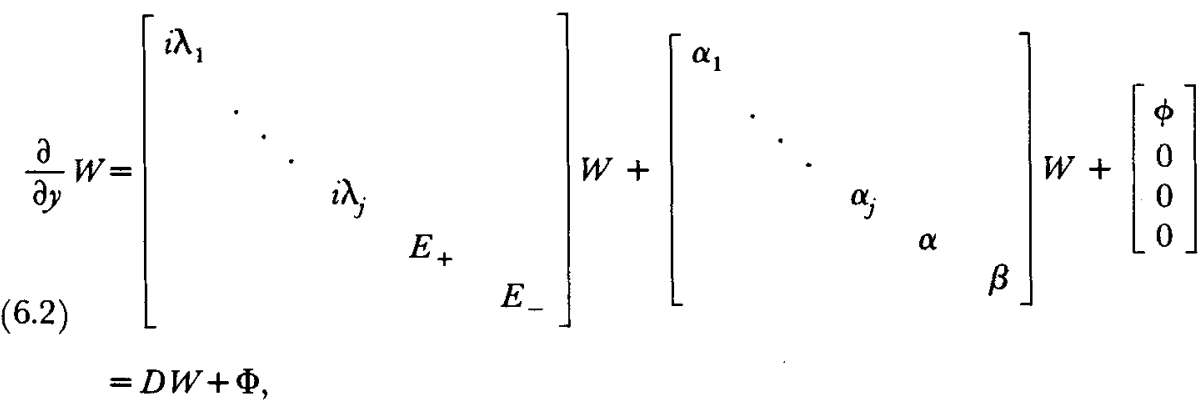

$$
\gamma W(0)=g
$$

with $g$ some smooth function. This is possible because the system $W^{\mathrm{I}}(0), W^{\mathrm{III}}(0)$, and $\gamma W(0)$ given is elliptic on $\mathfrak{A}_{1}$; and thus we can solve for $W(0)$ on $\mathfrak{U}_{1}$, modulo a smooth function, and extend so that $W F(W(0))$ is contained in $\tilde{\mathfrak{A}}_{1}$. Then $W$ is obtained by solving a hyperbolic equation for $W^{\text {II }}$ and a parabolic equation for $W^{\mathrm{IV}}$.

For $y \in\left[0, y_{1}\right]$ and $t \in\left(\tau_{1}-\epsilon, \tau_{1}+\epsilon\right)$, let $v_{\nu}=U^{-1}(1+K)^{-1} W=V^{-1} W$. Then $\beta v_{\nu}(0)=V^{-1} \gamma W(0)$ and $\partial / \partial y v_{\nu}=V^{-1} D V v_{\nu}+\left(\partial / \partial y V^{-1}\right) W+V^{-1} \Phi$. But by the construction of Sections 1-3, $D=V G V^{-1}+V_{y} V^{-1} \bmod \mathrm{PS}(-\infty)$ on $\tilde{\mathfrak{U}}_{1}$ for $y \in$ $\left[0, y_{1}\right]$, which implies that, modulo $C^{\infty}, \partial / \partial y v_{\nu}=G v_{\nu}$ on $\tilde{\mathfrak{X}}_{1}$ for $y \in\left[0, y_{1}\right]$. Now by construction, for $y \in\left(0, y_{1}\right], t \in\left(\tau_{1}-\epsilon, \tau_{1}+\epsilon\right),(y, x, t, \eta, \xi, \tau) \in \mathrm{WF}\left(v_{\nu}\right)$ implies $(x, t, \xi, \tau) \in \tilde{\mathfrak{A}}_{1}$. Also for $t \in\left(\tau_{1}-\epsilon, \tau_{1}\right), v_{\nu}-u_{\nu}$ is smooth. Thus $v_{\nu}$ can be smoothly extended to $\left(0, \tau_{1}+\epsilon\right) \times \Omega$ in such a fashion that $(\boldsymbol{\alpha})-(\boldsymbol{\delta})$ are satisfied. The proof is complete.

There are many ways to generalize this to higher-order systems; we just give one here. The main reason for mentioning it is that we want to relax the Kreiss-type condition, which, as remarked in Section 5, is not necessary for ellipticity of the system (3.5). The class we describe in the next theorem includes the Neumann problem for the wave equation, the boundary value problems (5.9) and (5.10) obtained from Maxwell's equations, and the system (5.11)-(5.12) obtained from transmission problems. The proof goes like that of Theorem 6.1 , with the check that (3.5) is elliptic left to the reader.

ThEOREM 6.2. Suppose $u=\left(u_{1}, \cdots, u_{k}\right)$ solves a second-order system

$$
\begin{array}{ll}
\frac{\partial^{2}}{\partial t^{2}} u_{j}-A_{j} y_{j}=0, & j=1, \cdots, k \\
\alpha(\nabla u)+\beta u=0 & \text { on } \partial \mathcal{O},
\end{array}
$$


where each $A_{j}$ is a second-order scalar elliptic operator on $\theta$ such that $\left[\begin{array}{lll}A_{1} & & \\ & & \\ & \\ & A_{k}\end{array}\right]$ defined by the boundary condition (6.4) differs from a negative selfadjoint operator by an operator of order one. Assume the boundary condition coercive. If $u$ is smooth near $\partial \theta$ for $t \in(0, \tau)$ and satisfies the non-grazing hypothesis, then conclusions (a) and (b) of Theorem 6.1 hold.

Here we think of the rays of each $D_{l}^{2}-A_{j}$ being propagated separately, only interacting on $\partial \Omega$. It is not necessary that (6.3) form a strictly hyperbolic system, since the various second-order wave equations are already completely decoupled.

It should be pointed out that Theorems 6.1 and 6.2 are sharper than Theorem 3.2 , even in the interior of $\Omega$. For example, if $u$ solves the wave equation $\left(\partial^{2} / \partial t^{2}-\Delta\right) u=0$ on the exterior of a region $K$ as presented in Figure 1, and if, for $t<0, u$ has a singularity only along the ray $\gamma_{1}$, then for $t>0$ we know that $u$ only has a singularity along $\gamma_{2}$, but from Theorem 3.2 one could not deduce that $u$ does not have a singularity along $\gamma_{3}$, which grazes $K$.

For a single wave equation $\left(\partial^{2} / \partial t^{2}-A\right) u=0$, any non-grazing ray hitting $\partial \Omega$ gives rise to a single non-grazing reflected ray. If $u$ has a singularity only along such a ray $\gamma$, this singularity propagates only along such a ray, reflected each time it hits $\partial \Omega$. We can take $u \notin H^{1}$ along $\gamma$ to start with, i.e., $u$ has infinite energy, and then $u \notin H^{1}$ all along $\gamma$. If the boundary conditions and the coefficients of $A$ are independent of $t$, we can smooth $u$ out a little by convoluting with a highly localized smooth function of $t$, obtaining a solution to the given boundary value problem for $\left(\partial^{2} / \partial t^{2}-A\right) v=0$, whose energy remains close to $\gamma$ for as long a time as desired. This generalizes the construction of Ralston in [9].

If $\theta$ is strictly bicharacteristically convex, then Theorem 6.2 yields very sharp results for solutions to a scalar wave equation. In fact, any solution $u$ smooth near

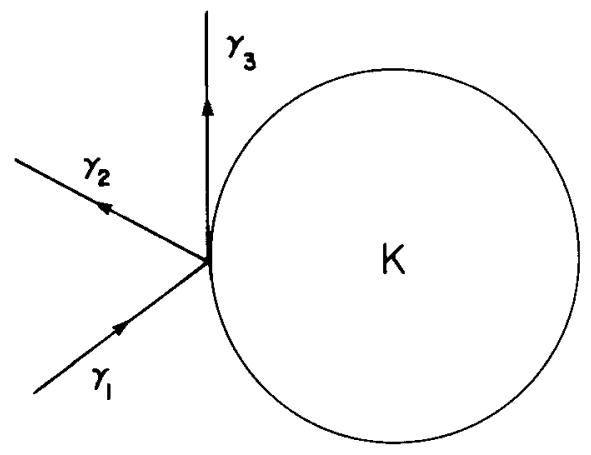

Figure 1. 
$\partial \Omega$ for small $t$ satisfies the non-grazing hypothesis, so conclusions (a) and (b) apply. An example is the Riemann function, solving $\left(\partial^{2} / \partial t^{2}-A\right) R=0$, the appropriate boundary condition, in a weak sense, and the initial value problem $R(0)=0, \partial / \partial t R(0)=\delta_{p}$, where $p$ belongs to the interior of $\theta$. This generalizes the results of Povzner and Sukharevskii [7].

In the higher-order case things are not so simple, as we shall illustrate in a moment. But first we give an example to show that the full story has not been told, for scalar second-order equations, even in the convex case.

In the following example, $\theta$ will be a strictly convex bounded region in $\mathbb{R}^{n}$, with smooth boundary, and $u$ will be a solution to the wave equation in $\mathbb{R} \times \theta$ with Dirichlet boundary conditions on $\partial \mathcal{O}$ :

$$
\begin{aligned}
\left(\frac{\partial^{2}}{\partial t^{2}}-\Delta\right) u & =0, \\
u & =0 \text { on } \partial \mathcal{O} .
\end{aligned}
$$

Suppose $u$ is smooth on the interior of $\mathbb{R} \times \theta$; must $u$ be smooth up to the boundary? The answer is no, and we proceed to construct an example. A related phenomenon has been studied by Keller and Rubinow [3] when $\theta$ is an elliptical domain in the plane.

To begin our construction, pick points $x_{j} \in \theta$ tending to a point $p_{0} \in \partial \theta$, and rays issuing from $x_{j}$, pointing parallel to a given tangent to $\partial \hat{\theta}$ at $p_{0}$. We suppose these rays, $\gamma_{j}$, travel in straight lines at unit speed and bounce off $\partial \theta$ according to the usual rule : angle of incidence equals angle of reflection. Exhaust $\theta$ by open sets $\theta_{1} \subset \subset \theta_{2} \subset \subset \cdots$ with the property that $\gamma_{j}$ lies in $\theta \backslash \theta_{j}$ for a unit time interval.

Define functions $\phi_{j} \in L_{\text {comp }}^{2}(\theta)$ with wave front set consisting of just one ray lying over $x_{j}$ such that $\phi_{j} \notin H^{1}(\theta)$, i.e., $\phi_{j}$ has infinite energy, and the solution $v_{j}$ to the mixed problem

$$
\begin{aligned}
\left(\frac{\partial^{2}}{\partial t^{2}}-\Delta\right) v_{j} & =0, \quad v_{j}=0 \text { on } \partial \theta, \\
v_{j}(0) & =\phi_{j}, \quad \frac{\partial}{\partial t} v_{j}(0)=0,
\end{aligned}
$$

has a singularity just along $\gamma_{j}$ in the interior of $\mathcal{O}$. That this can be done for small time follows from [1], and that it will hold for all time follows from reflection of singularities. In fact, the result of Lax and Nirenberg [6] suffices for the case considered here, but this example can be generalized. We also suppose $\operatorname{supp} \phi_{j}$ belongs to a small neighborhood $\mathscr{2}_{j}$ of $x_{j}$. 
Now we will want to smooth $\phi_{j}$ out just slightly and perhaps multiply by a small constant, say

$$
u_{j}=c_{j} \int \rho_{j}(\tau) v_{j}(t-\tau) d \tau,
$$

where $\rho_{j} \in C_{0}^{\infty}(\mathbb{R})$ has small support and $c_{j}$ is chosen so that

$$
\begin{aligned}
\sum_{|\alpha| \leq j} \int_{0}^{1} \int_{\mathcal{O}_{j}}\left|D^{\alpha} u_{j}\right|^{2} d x d t & \leqq 2^{-j} \\
\int_{\mathscr{Q}_{j}}\left|\nabla u_{j}(x, 0)\right|^{2} d x & \geqq 10^{j}\left(1+\sum_{k<j} \int_{\mathcal{O}}\left|\nabla u_{k}(x, 0)\right|^{2}\right) \\
\left\|u_{j}(x, 0)\right\|_{L^{2}(\mathcal{O})} & \leqq 2^{-j}
\end{aligned}
$$

Let $u=\sum_{j=1}^{\infty} u_{j}$. By (6.6) and (6.8), $u \in C^{\infty}((0,1) \times \theta)$ and solves the mixed problem (6.5) there, in a weak sense. It follows that $u$ continues to solve such a mixed problem for all time, and by reflection of singularities, $u$ must be smooth on the interior of $\mathbb{R} \times \theta$. However, $u(0)$ has infinite energy, by (6.7), so $u$ cannot be smooth up to the boundary.

In this example, one can see that the singularity of $u$ on the boundary travels along a curve that is trying to be a ray, except that it is trapped on $\mathbb{R} \times \partial \theta$. One might speculate that boundary singularities of solutions to boundary value problems propagate along such "tangential bicharacteristics" in general. I would not venture to say whether this problem is as difficult as the general glancing ray problem, but the phenomenon described above seems not to be well understood at the present time.

Finally, we give an example of how things can be even more mysterious in the case of higher-order systems. The phenomenon we consider is that $u$ solves a hyperbolic mixed system and has a singularity along a single ray $\gamma_{0}$, for $t<t_{0}$, which hits $\partial \Omega$, passing over $p_{0} \in T^{*}(\partial \Omega) \backslash 0$. In the general case, several reflected rays could exist, and at least one of them could be grazing. In such a case, Theorems 3.2, 4.2, 6.1, and 6.2 would not apply. To take a particular example, let $u$ solve a transmission problem

$$
\begin{aligned}
& \left(\frac{1}{c^{2}} \frac{\partial^{2}}{\partial t^{2}}-\Delta\right) u=0 \text { in } \mathcal{\Theta}_{1} \\
& \left(\frac{\partial^{2}}{\partial t^{2}}-\Delta\right) u=0 \text { in } \mathbb{R}^{2} \backslash \mathcal{O}_{1}
\end{aligned}
$$


$\theta$, being some convex region in $\mathbb{R}^{2}$ with smooth boundary. The boundary conditions are that $u$ and $\nabla u$ be continuous across $\partial \theta_{1}$. We suppose $c>1$, i.e., sound speed is greater than one in $\theta_{1}$. Then, as mentioned in Section 5 , a ray coming in from $\mathbb{R}^{2} \backslash \theta_{1}$ and hitting $\partial \Theta_{1}$ too obliquely produces a singularity along the reflected ray, but no singularity going into $\theta_{1}$.

A ray just on the other side of the critical ray, produces reflected and refracted rays as indicated in Figure 2. If $u$ is in $L^{2}$ along the ray $\gamma_{1}$ but not in $H^{1}$, then $u$ has the same property along all the rays indicated in Figure 2.

Therefore, if we consider a sequence of such rays approaching a critical ray $\gamma_{0}$, then a simple modification of the construction in the previous example will produce an example of a solution $u$, with a singularity only along the ray $\gamma_{0}$ for $t<t_{0}$, which spews off a two-dimensional sheet of singularities for $t>t_{0}$.

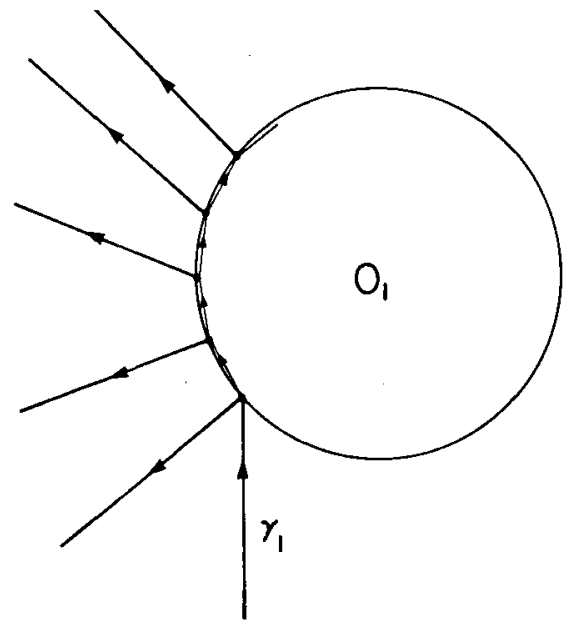

Figure 2.

\section{Appendix}

A Technicality

In this appendix we take care of minor problems caused by the fact that a smooth family of pseudo-differential operators of order one on $\partial \Omega, K=K\left(y, x, D_{x}\right)$, is not a pseudo-differential operator on $\Omega=\left(0, y_{0}\right) \times \partial \Omega$. Fortunately, $K$ acts like a pseudo-differential operator on the types of functions and distributions we are interested in. Note that

$$
K f(y, x)=(2 \pi)^{-n-1} \int e^{-i(y \eta+x \cdot \xi)} K(y, x, \xi) \hat{f}(\eta, \xi) d \eta d \xi,
$$

where

$$
\hat{f}(\eta, \xi)=\int e^{i(\eta \eta+x \cdot \xi)} f(y, x) d y d x
$$


Thus the only thing that prevents $K$ from being a pseudo-differential operator of order one on $\Omega$ is that its symbol $\sigma_{K}(y, x, \eta, \xi)=K(y, x, \xi)$ fails to be smooth along the rays $(y, x, \eta, 0)$, where $\xi=0$.

We could alter $\sigma_{K}$ in a small conic neighborhood $\Gamma$ of such rays to be smooth, obtaining $L \in \mathrm{PS}(1)$ and if $\hat{f}$ were rapidly decreasing in a neighborhood of $\Gamma$, then $K f-L f \in C^{\infty}$, by (A.1). We have proved the following:

LemmA A.1. If $\Gamma$ is a conic neighborhood in $T^{*} \Omega \backslash 0$ of the set $\Sigma$ of rays $(y, x, \eta, 0)$, then there is an $L \in \mathrm{PS}(1)$ such that $K f-L f \in C^{\infty}$ for all $f$ with $\mathrm{WF}(f) \cap \Gamma=\varnothing$.

This lemma is given meaning by the following:

Lemma A.2. If $(\partial / \partial y-K) u \in C^{\infty}$, then $\operatorname{WF}(u) \cap \Sigma=\varnothing$.

Note that elements of $\Sigma$ are not characteristics of $\partial / \partial y-K$, so if this were a pseudo-differential operator the conclusion would be well known. The difficulty is that the "symbol" of $\partial / \partial y-K$ is singular precisely on $\Sigma$. Actually, in practice such first-order systems as one treats are either differential equations or arise from reducing higher-order systems of differential equations, in which case the fact that $\mathrm{WF}(u) \cap \Sigma=\varnothing$ would be a simple consequence of Lemma A.1, but for the sake of completeness we prove this result.

Proof of Lemma A.2: We need to show that, for each $\left(x_{0}, y_{0}\right) \in \Omega$,

$$
\left\langle\alpha e^{i \lambda(y+\xi \cdot x)}, u\right\rangle=O\left(|\lambda|^{-N}\right) \text { as }|\lambda| \rightarrow \infty
$$

uniformly for $\xi$ small, $\alpha$ belonging to a compact set of test functions with support in a small neighborhood of $\left(x_{0}, \xi_{0}\right)$, for each $N$. Suppose we have

$$
\left\langle\alpha e^{i \lambda(y+\xi \cdot x)}, u\right\rangle=O\left(|\lambda|^{s}\right) \quad \text { as } \quad|\lambda| \rightarrow \infty
$$

uniformly with respect to such parameters, for some $s \in R$. Now

$$
\left\langle\alpha e^{i \lambda(y+\xi \cdot x)},\left(K-\frac{\partial}{\partial y}\right) u\right\rangle=O\left(|\lambda|^{-N}\right)
$$

hence

$$
\begin{aligned}
\left\langle e^{i \lambda y} K^{*}\left(\alpha e^{i \lambda \xi \cdot x}\right)\right. & \left.+i \lambda \alpha e^{i \lambda(y+\xi \cdot x)}, u\right\rangle \\
= & -\left\langle\frac{\partial \alpha}{\partial y} e^{i \lambda(y+\xi \cdot x)}, u\right\rangle+O\left(|\lambda|^{-N}\right)=O\left(|\lambda|^{s}\right) .
\end{aligned}
$$

$K^{*}=K^{*}\left(y, x, D_{x}\right)$ is a smooth family of pseudo-differential operators on $\partial \Omega$, so we 
have an asymptotic expansion

$$
e^{-i \lambda \xi \cdot x} K^{*}\left(\alpha e^{i \lambda \xi \cdot x}\right)=|\lambda| K_{1}^{*}(y, x, \pm \xi) \alpha+\cdots
$$

and the remainder belongs to a precompact subset of $C_{0}^{\infty}(\Omega)$ if $\alpha$ does. Hence

$$
\left\langle\left(i \pm K_{1}^{*}(y, x, \pm \xi)\right) \alpha e^{i \lambda(y+\xi \cdot x)}, u\right\rangle=O\left(|\lambda|^{s-1}\right) .
$$

Since $K_{1}^{*}$ is homogeneous of degree one in $\xi$, we can pick $|\xi|<\delta$ such that $\left\|K_{1}^{*}(y, x, \xi)\right\|<\frac{1}{2}$, and if $\alpha=\left(i \pm K_{1}^{*}(y, x, \pm \xi)\right)^{-1} \beta$, then $\alpha$ belongs to a precompact subset of $C_{0}^{\infty}(\Omega)$ for $|\xi|<\delta$, provided $\beta$ does. Therefore (A.3) implies

$$
\left\langle\beta e^{i \lambda(y+\xi \cdot x)}, u\right\rangle=O\left(|\lambda|^{s-1}\right) \text { as }|\lambda| \rightarrow \infty,
$$

uniformly with respect to precompact sets of $\beta$ and small $\xi$, an improvement over (A.2). An inductive argument finishes the proof. (Note that no further shrinking of the neighborhood of $\Sigma$ is required.)

\section{Bibliography}

[1] Hörmander, L., On the existence and regularity of solutions of linear pseudo-differential equations, L'Enseignement Math., 1971, pp. 99-163.

[2] Hörmander, L., Fourier integrals operators, I, Acta Math. 127, 1971, pp. 79-183.

[3] Keller, J., and Rubinow, S., Asymptotic solutions of eigenvalue problems, Annals of Physics, 9, 1960, pp. 24-75.

[4] Kreiss, H., Initial boundary value problems for hyperbolic systems, Comm. Pure Appl. Math., 23, 1970, pp. 277-298.

[5] Majda, A., and Osher, S., Reflection of singularities at the boundary, Comm. Pure Appl. Math., this issue, pp. $479-499$.

[6] Nirenberg, L., Lectures on linear partial differential equations, Regional Conference Series in Math., No. 17, Providence, R. I., 1973.

[7] Povzner, A., and Sukharevskii, J., Discontinuities of the Green's function of a mixed problem for the wave equation, Mat. Sburnik, Akad. Nauk, 51, 1960, pp. 3-26; Amer. Math. Soc. Translations, Ser. 2, Vol. 47, pp. 131-156.

[8] Polking, J., Boundary value problems for parabolic systems of partial differential equations, Proc. Symp. Pure Math., Vol. X, Providence, R. I., 1967.

[9] Ralston, J., Solutions of the wave equation with localized energy, Comm. Pure Appl. Math., 22, 1969, pp. 807-824.

[10] Rauch, J., and Massey, F., Differentiability of solutions to hyperbolic initial-boundary value problems, Trans. Amer. Math. Soc., 189, 1974, pp. 303-318.

[11] Taylor, M., Pseudo-Differential Operators, Springer Lecture Notes Series, 1974.

Received January, 1975. 